\title{
No evidence for interstellar planetesimals trapped in the Solar system
}

\author{
A. Morbidelli ${ }^{\oplus},{ }^{1 \star}$ K. Batygin,${ }^{2 \star}$ R. Brasser ${ }^{3}$ and S. N. Raymond ${ }^{4 \star}$ \\ ${ }^{1}$ Laboratoire Lagrange, UMR 7293, Université de Nice Sophia-Antipolis, CNRS, Observatoire de la Côte d'Azur, Boulevard de l'Observatoire, F-06304 Nice \\ Cedex 4, France \\ ${ }^{2}$ Division of Geological and Planetary Sciences, California Institute of Technology, Pasadena, CA 91125, USA \\ ${ }^{3}$ Earth Life Science Institute, Tokyo Institute of Technology, Ookayama, Meguro-ku, Tokyo 152-8550, Japan \\ ${ }^{4}$ Laboratoire d'Astrophysique de Bordeaux, Université de Bordeaux, CNRS, Allée Geoffroy Saint-Hilaire, F-33165 Pessac, France
}

Accepted 2020 June 8. Received 2020 June 8; in original form 2020 May 5

\begin{abstract}
In two recent papers published in MNRAS, Namouni and Morais claimed evidence for the interstellar origin of some small Solar system bodies, including: (i) objects in retrograde coorbital motion with the giant planets and (ii) the highly inclined Centaurs. Here, we discuss the flaws of those papers that invalidate the authors' conclusions. Numerical simulations backwards in time are not representative of the past evolution of real bodies. Instead, these simulations are only useful as a means to quantify the short dynamical lifetime of the considered bodies and the fast decay of their population. In light of this fast decay, if the observed bodies were the survivors of populations of objects captured from interstellar space in the early Solar system, these populations should have been implausibly large (e.g. about 10 times the current main asteroid belt population for the retrograde co-orbital of Jupiter). More likely, the observed objects are just transient members of a population that is maintained in quasi-steady state by a continuous flux of objects from some parent reservoir in the distant Solar system. We identify in the Halley-type comets and the Oort cloud the most likely sources of retrograde co-orbitals and highly inclined Centaurs.
\end{abstract}

Key words: chaos - diffusion-methods: numerical - celestial mechanics - planets and satellites: dynamical evolution and stability.

\section{INTRODUCTION}

The passages of the interstellar objects 1I/Oumuamua and 2I/Borisov through the Solar system on clearly hyperbolic orbits have stimulated interest in extrasolar planetesimals and their similarities and differences with the small bodies of the Solar system. It is therefore not surprising that the claims by Namouni \& Morais $(2018,2020)$ on the existence of populations of extrasolar planetesimals stranded in the Solar system since 4.5 Gy ago have attracted some attention in the astronomical community and in the media. Although the willingness of Namouni and Morais to consider unconventional possibilities is admirable, the analyses outlined in the aforementioned papers are not correct. In particular, the logic of the presented arguments suffers from significant drawbacks, and the methods are unsupported by modern knowledge of the behaviour of chaotic dynamical systems. Below we summarize the main steps of the Namouni and Morais analysis, and then discuss why they are not valid. kbatygin@caltech.edu

$(\mathrm{KB})$;

\section{A BRIEF SUMMARY OF THE WORK BY NAMOUNI AND MORAIS}

In their 2018 paper, Namouni and Morais consider the object (514107) 2015 BZ509 that is currently on a retrograde orbit, executing co-orbital motion with Jupiter. In their 2020 paper, they extend their analysis to several other small bodies in co-orbital resonances with the giant planets and to Centaurs with highly inclined or retrograde orbits. These are the objects that they claim to be of interstellar origin.

Their work can be very simply summarized as follows:

(i) They clone the observed objects about a million times (the exact number depends from object to object), with orbital elements sampling the current uncertainty on the nominal orbits of the real objects.

(ii) They integrate all of the clones backwards in time for $4.5 \mathrm{~Gy}$ (the approximate age of the Solar system).

(iii) They find that the vast majority of the clones do not survive for the whole integration time-span. Most are ejected from the Solar system, or collide with the Sun or the planets. The typical dynamical lifetimes are a few Myr. Only one clone in a million of (514107) 2015 BZ509 preserves its initial orbital characteristics for 4.5 Gy and only $\sim 1.5 \times 10^{-4}$ of the clones of all objects [with the exception 
of $2008 \mathrm{KV} 42$ and (471325) $\left.2011 \mathrm{KT}^{2}\right]^{1}$ are still on orbits bound to the Sun at the end of the integration time-span. Hereafter, we refer to these as the ' 4.5 Gy surviving trajectories'.

(iv) They then invoke a very personal view of the Copernican principle - according to which we should not be living in a special moment of the history of the Solar system (Bondi 1961) - to assert that the real objects must have followed the 4.5 Gy surviving trajectories, even if these are just a strict minority of all possible dynamical outcomes. Otherwise - the authors claim - we must be living in a special moment when these objects are observable, which would be only a small fraction of the Solar system lifetime.

(v) From this, they conclude that the considered objects must be of interstellar origin. They justify their claim with the following argument. The clones following the 4.5 Gy surviving trajectories are in the end on highly inclined orbits with respect to the plane of the planets, whereas at that time the whole Solar system should have been shaped like a disc (namely, all inclinations should have been small). The considered objects must therefore have originated outside our system, i.e. from elsewhere in the Galaxy.

We now discuss the problems with this approach.

\section{THE FATAL FLAWS}

Although the classical systems studied in celestial mechanics conserve energy and momentum, the dream of Laplace to know the past history of a system by integrating its evolution backwards in time with sufficient precision cannot be fulfilled. The reason is that, as demonstrated by Poincaré (1899), the systems of celestial mechanics are in general non-integrable; most of the initial conditions lead to chaotic dynamics (Henon \& Heiles 1964). This is certainly the case for the objects considered by Namouni \& Morais (2018, 2020), given their close encounters with the planets and statistically short dynamical lifetimes. A swarm of particles on chaotic trajectories can be described with the tools of statistical mechanics. In particular, their entropy (the exponential of which in this case is related to the phase-space volume occupied by the ensamble of clones) increases with time (Gibbs 1902). Because of the exponential accumulation of errors in the presence of chaotic dynamics, the entropy increases in both forward and backward integrations (Gaspard 2005). Therefore, backward and forward integrations are statistically equivalent. The backward integrations do not reproduce - even in a statistical sense - the real past evolution of the system because, if one could follow the real evolution backward in time, the entropy of the system would decrease in agreement with the second law of thermodynamics.

For clarification, consider the following thought experiment. Suppose there is a bottle full of aromatic molecules in a room; the cap is opened and the aromatic molecules diffuse out of the bottle into the room. Simulating this system would not be difficult; the diffusion of the molecules from the bottle into the room corresponds to a net increase in entropy. Now, imagine to come into the room once its air is full of aromatic molecules and to wonder where they come from. The bottle is open in one corner of the room and you wonder whether the aromatic molecules may have come from there. Given that you believe yourself to be the incarnation of Laplace's demon, you measure all the positions and velocities of the aromatic molecules and of all other gas molecules in the room

${ }^{1}$ The clones of 2008 KV42 and (471325) 2011 KT19 have a higher survival rate and these objects are discussed separately in Section 3. and you start simulating their dynamical evolution backwards in time. Given that no measurement is made with infinite precision, the aromatic molecules will never appear to go back all together into the bottle. Therefore, you would conclude - incorrectly - that the bottle was not the source of the perfume. Now, the objects considered in this discussion are like the aromatic molecules and the Solar system's primordial disc is like the bottle. Then, it should not be surprising that the 4.5 Gy surviving trajectories in the simulations by Namouni and Morais are not found in the disc.

This issue is well known by all dynamical astronomers. Nobody has ever seriously thought to find the source regions of nearEarth asteroids, Jupiter-family comets, or long-period comets by integrating their evolution backwards in time. Instead, state-ofthe-art models for these populations make an educated guess of their respective source regions, then simulate forward in time the evolution of the objects, and finally compare the results with the observations validating, in case of success, the initial ansatz on the source (see Bottke et al. 2002 and Granvik et al. 2018 for the nearEarth asteroids; Levison \& Duncan 1997 and Nesvorny et al. 2017 for the Jupiter-family comets; and Wiegert \& Tremaine 1999 for the long-period comets).

The second serious flaw is in the application of the Copernican principle. The Solar system is an evolving system and therefore there is no reason a priori that the Solar system that we see today is identical to the Solar system in the past. A strict application of the principle as interpreted by Namouni and Morais would simply lead to the statement that the Solar system has always been like it is now. Indeed, such a view is sure to cause intellectual discomfort to anyone who has ever seen a shooting star (a meteor) zoom across the night sky, only to disappear for all time. Moreover, followed to its logical conclusion, this would imply that all objects out of the Solar system's mid-plane - including Pluto and even Mercury - must be exogenous! Furthermore, notice that the Copernican principle as stated in Namouni \& Morais (2020) is contradicted within the paper itself. In fact, the $4.5 \mathrm{~Gy}$ surviving trajectories lead the objects on to radically different orbits than those they occupy today. So, we would be living in a special time to see them on their current orbits.

Leaving behind this philosophical discussion, the truth is that if the dynamical lifetime of a set of observed objects is short, the objects can be the relic of a primordial population only if the latter originally comprised many more bodies. This is, for instance, the case of the scattered disc in the trans-Neptunian population: the scattered disc objects are unstable and they are believed to be the remnant of a primordial scattered disc that was originally $\sim 100$ times more populated than now (Duncan \& Levison 1997), formed during the period of Neptune's migration (Brasser \& Morbidelli 2013; Nesvorny et al. 2017). With a probability of one in a million to remain on its current trajectory for $4.5 \mathrm{~Gy}$, (514107) 2015 BZ509 should therefore be the remnant of an initial population of about one million objects of comparable size (about 3-4 km in diameter) on similar orbits (retrograde, with semimajor axis oscillating around Jupiter's value; Meeus 2019), i.e. about 10 times the current population in the asteroid belt. This is quite implausible given the expected volume density of interstellar planetesimals in the galaxy (Meech et al. 2017; Do, Tucker \& Tonry 2018).

The third fatal flaw of Namouni and Morais is to neglect a priori the possibility that these strongly unstable objects are transient representatives of a population that is maintained in steady state. Indeed, to reconcile short dynamical lifetimes of real objects with 
the Copernican principle, a steady-state scenario is the most likely solution. There are multiple examples of populations of small bodies in the Solar system with individually short lifetimes that are maintained in steady state by a flux of new objects from a parent reservoir. The near-Earth asteroid population is a clear example. With a median lifetime of $\sim 10 \mathrm{Myr}$ (Gladman et al. 1997), the individual near-Earth asteroids come and go in the blink of an eye compared to the Solar system's age, but are substituted by new objects leaking out of the main asteroid belt (e.g. Morbidelli \& Vokrouhlicky 2003). The co-orbital asteroids of the Earth or Venus have a very short residence time ( $\sim 25000 \mathrm{yr})$ on their characteristic orbits, but their populations as a whole are kept in steady state by the temporary trapping of near-Earth asteroids (Morais \& Morbidelli 2002, 2006). The Jupiter-family comets have a combined dynamical/physical lifetime of $\sim 10^{4} \mathrm{yr}$ (Levison \& Duncan 1997), but their population is kept in steady state by the injection of objects that originate within the scattered disc (Duncan \& Levison 1997). The long-period comets have a lifetime of a few orbital revolutions, but are kept in steady state as a population by the incoming flux of new comets from the Oort cloud (Wiegert \& Tremaine 1999). So, a steady-state scenario should be the default explanation for the existence of short-lived small bodies and one should look for more exotic explanations only if no source capable of maintaining the required steady state is found.

It is not the purpose of this short rebuttal to build steady-state models for the objects considered in the Namouni and Morais papers. Nevertheless, a few suggestions can be provided. For (514107) 2015 BZ509, the retrograde co-orbital of Jupiter, the population of Halley-type comets (HTCs; themselves coming from the Oort cloud; Fernandez \& Gallardo 1994) could be an obvious source to consider. The inclination of (514107) 2015 BZ509 is well within the range of HTCs and it is well known that during their dynamical evolution short-period comets are often temporarily trapped in mean motion resonances with the giant planets. For the highly inclined or retrograde Centaurs, the obvious source would be the Oort cloud, from which objects come into the inner Solar system with an isotropic distribution of inclinations (Brasser et al. 2012b). Here, a difficulty is that the Centaurs have semimajor axes much smaller than those of the typical comets from the Oort cloud; encounters with the planets can decrease the semimajor axes, but if the objects encounter only Uranus and Neptune at high relative velocity, as it is the case for the considered highly inclined Centaurs, the planetary close encounters may not be very effective. Nesvorny et al. (2019) indeed found a deficit of highly inclined Centaurs in their model, although this may be an issue of small number statistics (one of such objects was found in the survey, while the model predicts a 10 per cent probability of having one detection). Nevertheless, the issue needs further analysis. As an alternative explanation, Gomes, Soares \& Brasser (2015) and Batygin \& Brown (2016) proposed that the highly inclined Centaurs are one of the signatures of the existence of a putative ninth planet in the distant Solar system.

The objects 2008 KV42 and (471325) 2011 KT19 may require specific consideration. With a median dynamical lifetime of 100 $200 \mathrm{Myr}$ and a 4.5 Gy survival probability of 15 percent in the simulations of Namouni \& Morais (2020), these objects may in principle be the remnant of an initially large, but not abnormal population, possibly established during the dispersal of the original planetesimal disc in the presence of a natal stellar cluster, which is the scenario invoked for Sedna and the inner Oort cloud (Brasser et al. 2012a). As an alternative, Batygin et al. (2019) reproduced their existence (called Niku and Drac in that publication) under the Planet IX hypothesis. Clearly, more investigations are required before we can conclude on the origin of these objects. Nevertheless, their capture from interstellar space is far from obvious. In fact, putative objects trapped from the interstellar space are expected to have orbits typical of the Oort cloud (Levison et al. 2010; Hands \& Dehnen 2020), i.e. radically different from those of 2008 KV42 and (471325) 2011 KT19. $^{2}$ In the end, Namouni and Morais do not present any model reproducing the orbits of these objects (or any other Centaur) via the capture of interstellar bodies, meaning that even the basic premise of this scenario remains undemonstrated.

\section{CONCLUSION}

We have discussed in some detail the fatal flaws that invalidate the claims made in Namouni \& Morais $(2018,2020)$ pertaining to the existence of extrasolar planetesimals on bound Solar system orbits. Although it is not strictly impossible that interstellar comets can become temporarily or even permanently trapped within the Solar system, to date, no evidence for their existence has been marshaled. Thus, to study exotic planetesimals our attention can only turn to 1I/Oumuamua and 2I/Borisov and to the other objects on hyperbolic trajectories that will undoubtedly be discovered in large numbers in the future.

\section{REFERENCES}

Batygin K., Brown M. E., 2016, ApJ, 833, L3

Batygin K., Adams F. C., Brown M. E., Becker J. C., 2019, Phys. Rep., 805,1

Bondi H., 1961, Cosmology. Cambridge Univ. Press, Cambridge

Bottke W. F., Morbidelli A., Jedicke R., Petit J.-M., Levison H. F., Michel P., Metcalfe T. S., 2002, Icarus, 156, 399

Brasser R., Morbidelli A., 2013, Icarus, 225, 40

Brasser R., Duncan M. J., Levison H. F., Schwamb M. E., Brown M. E., 2012a, Icarus, 217, 1

Brasser R., Schwamb M. E., Lykawka P. S., Gomes R. S., 2012b, MNRAS, 420, 3396

Do A., Tucker M. A., Tonry J., 2018, ApJ, 855, L10

Duncan M. J., Levison H. F., 1997, Science, 276, 1670

Fernandez J. A., Gallardo T., 1994, A\&A, 281, 911

Gaspard P., 2005, in Collet P., Courbage M., Métens S., Neishtadt A., Zaslavsky G., eds, NATO Science Series, Vol. 182, Dynamical Systems Theory of Irreversibility. Kluwer, Dordrecht, p. 107

Gibbs J. W., 1902, Elementary Principles in Statistical Mechanics: Developed with Especial Reference to the Rational Foundation of Thermodynamics. Charles Scribner's Sons, New York

Gladman B. J. et al., 1997, Science, 277, 197

Gomes R. S., Soares J. S., Brasser R., 2015, Icarus, 258, 37

Granvik M. et al., 2018, Icarus, 312, 181

Hands T. O., Dehnen W., 2020, MNRAS, 493, L59

Henon M., Heiles C., 1964, AJ, 69, 73

Levison H. F., Duncan M. J., 1997, Icarus, 127, 13

Levison H. F., Duncan M. J., Brasser R., Kaufmann D. E., 2010, Science, 329,187

Meech K. J. et al., 2017, Nature, 552, 378

Meeus J., 2019, J. Br. Astron. Assoc., 129, 170

${ }^{2}$ Siraj \& Loeb (2019) claimed that interstellar objects trapped in the Solar system by a Jupiter encounter can acquire highly inclined Centaurs orbits. Nevertheless, the orbits of 2008 KV42 and (471325) 2011 KT19 are more than $10 \sigma$ away from the maximum of the probability distribution of the orbital elements of their captured objects. In addition, Hands \& Dehnen (2020) reported that they could not reproduce Siraj and Loeb's results. 
Morais M. H. M., Morbidelli A., 2002, Icarus, 160, 1

Morais M. H. M., Morbidelli A., 2006, Icarus, 185, 29

Morbidelli A., Vokrouhlický D., 2003, Icarus, 163, 120

Namouni F., Morais M. H. M., 2018, MNRAS, 477, L117

Namouni F., Morais M. H. M., 2020, MNRAS, 494, 2191

Nesvorný D., Vokrouhlický D., Dones L., Levison H. F., Kaib N., Morbidelli A., 2017, ApJ, 845, 27

Nesvorný D. et al., 2019, AJ, 158, 132
Poincaré H., 1899, Les méthodes nouvelles de la mécanique céleste. Gauthier-Villars, Paris

Siraj A., Loeb A., 2019, ApJ, 872, L10

Wiegert P., Tremaine S., 1999, Icarus, 137, 84

This paper has been typeset from a $\mathrm{T}_{\mathrm{E}} \mathrm{X} / \mathrm{L} \mathrm{T} \mathrm{E} \mathrm{X}$ file prepared by the author. 